\title{
The Warburg effect then and now: From cancer to inflammatory diseases
}

Authors

Eva M. Palsson-McDermott,

Luke A. J. O'Neill

\begin{abstract}
Inflammatory immune cells, when activated, display much the same metabolic profile as a glycolytic tumor cell. This involves a shift in metabolism away from oxidative phosphorylation towards aerobic glycolysis, a phenomenon known as the Warburg effect. The result of this change in macrophages is to rapidly provide ATP and metabolic intermediates for the biosynthesis of immune and inflammatory proteins. In addition, a rise in certain tricarboxylic acid cycle intermediates occurs notably in citrate for lipid biosynthesis, and succinate, which activates the transcription factor Hypoxia-inducible factor. In this review we take a look at the emerging evidence for a role for the Warburg effect in the immune and inflammatory responses. The reprogramming of metabolic pathways in macrophages, dendritic cells, and T cells could have relevance in the pathogenesis of inflammatory and metabolic diseases and might provide novel therapeutic strategies.
\end{abstract}

Introduction

Metabolism is the process whereby biochemicals are turned over to generate energy (in the form of ATP) or are used in the synthesis of macromolecules. The major triumph of biochemistry in the 20th century was the unraveling of these complex pathways of anabolism (biosynthesis) and catabolism (the breaking down of molecules). More recently, metabolism has reemerged as a process to generate signaling molecules for multiple cellular responses. This most notably occurs in cancer but also in normal cell function, a good example here being epigenetic changes, which rely on acetylation and methylation reactions. The immune and inflammatory responses have also been shown to involve profound metabolic changes such that analysis of these responses is providing new insights into the complex processes of host defense and tissue restoration after injury.

In a normal resting cell, energy demands are met as glucose entering glycolysis is converted to pyruvate, which enters the tricarboxylic acid (TCA) cycle where it becomes oxidized to generate ATP. Under normoxic conditions one glucose molecule is oxidized by glycolysis, generating two molecules of pyruvate, which enter the mitochondria. Pyruvate is then decarboxylated by pyruvate dehydrogenase (PDH) into acetyl-CoA allowing it to enter the TCA cycle. The total energy gain under these conditions is roughly 36 ATP per one molecule of glucose. However, in a situation of hypoxia or anoxia a cell has the ability to divert pyruvate away from oxidative phosphorylation in its mitochondria, 
allowing for ATP generation during low oxygen. One glucose molecule will then still generate two molecules of pyruvate. However pyruvate will now be converted into lactate by LDH in the cytosol. This reaction generates only two molecules of ATP; but it allows for regeneration of NAD+, which is required for glycolysis to proceed. When cells transform into malignant rapidly growing tumor cells their demand for biosynthetic precursors and energy change. In order to meet these new requirements the cells change their metabolic profile from a comparatively low rate of glycolysis followed by oxidation of pyruvate by the TCA cycle, to a much lower rate of oxidative phosphorylation and a high rate of glycolysis followed by lactic acid production. This occurs under aerobic conditions, and the high glycolytic rate and high glucose dependency was termed the "Warburg effect", named after Otto Warburg who in 1923 first defined this switch in metabolism, and suggested it was a fundamental cause of cancer [1]. This glycolytic switch in cancer cells may provide an explanation for their extraordinary tolerance to extreme local hypoxia, or anoxia, and their capacity to compete with normal untransformed cells under normoxia.

As evidence starts to emerge showing how the high demands for biosynthetic precursors including proteins, lipids, and nucleic acids as well as the increased energy demand of cells responding to infection or inflammation are in fact also met by the same metabolic switch, a surge of interest into the field of metabolic regulation in inflammation has ensued.

The Warburg effect

Warburg and co-workers pioneered research on respiration and biosynthesis during the early 20th century [2]. In the 1920's he first presented evidence that cancer cells produced lactate from glucose under normoxic conditions $[1,3,4]$. Warburg also contributed to the field of photosynthesis. He described an inhibitory effect of $\mathrm{O} 2$ on photosynthesis whereby 02 acts as a competitive inhibitor of $\mathrm{CO} 2$ at the stage of carbon fixation which, combined with a stimulatory effect of 02 on photorespiration [5] is also known as the Warburg effect; however completely distinct from the aerobic glycolysis reviewed here.

The cytosolic pyruvate generated by glycolysis has two alternative routes (Fig. 1). As described above, when oxygen is plentiful pyruvate is taken up by the mitochondria where it enters the Kreb's cycle, also known as the TCA, or citric acid cycle. When a tissue must function anaerobically or during hypoxia, such as in the case of a short burst of muscular activity, pyruvate cannot be oxidized further, because of the lack of oxygen. Instead pyruvate gets reduced to form lactate.

Figure 1. 


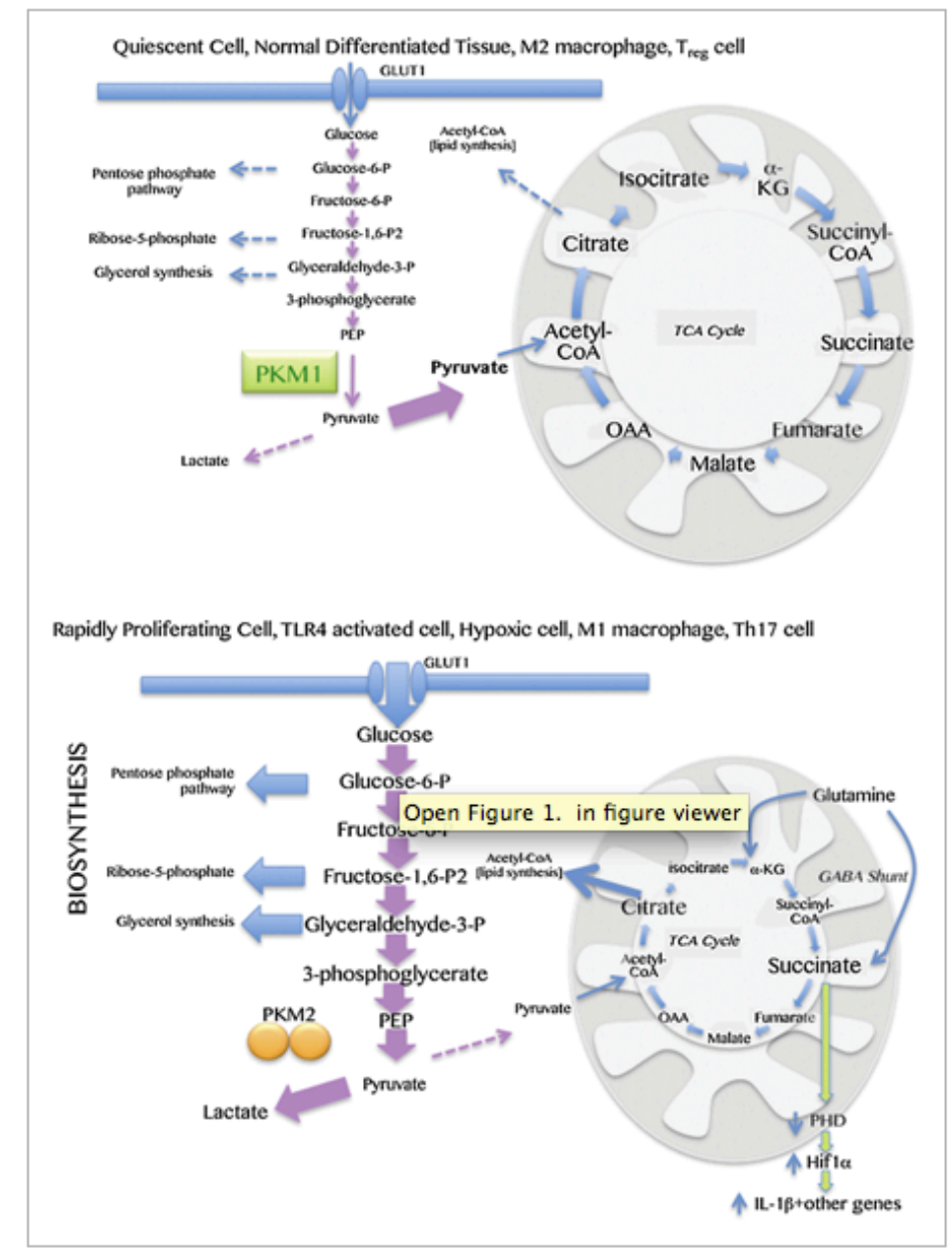

Schematic representation of metabolic pathways of normal resting cells, compared to activated immune cells or cancer cells. Top: Normal cells will generate ATP through a combination of glycolysis and mitochondrial respiration by the TCA cycle. Bottom: In highly proliferating cells such as tumor cells, or in activated M1 macrophages, or Th17 cells, ATP and essential components used in biosynthesis are generated by switching metabolism from oxidative phosphorylation to aerobic glycolysis, the so called "Warburg effect". Upregulated expression of GLUT1 allows for an increased uptake of glucose. An increased expression of PKM2 will cause pyruvate to convert to lactate diverting metabolism away from the mitochondria. Intermediates of glycolysis are allowed to build up, meeting the high-energy demands, and increased biomass requirements of cell proliferation. These cells also display an increase in succinate originating from glutaminolysis via $\alpha$-ketoglutarate, together with an upregulated GABA shunt.

In order to support proliferation and the increased biosynthetic demands, rapidly growing cancer cells make use of this hypoxic reaction and while doing so develop a great appetite for glucose (Fig. 1). As glucose is broken down to pyruvate, intermediates of glycolysis are used for nucleotide and amino acid synthesis as well as for NADPH production through the pentose phosphate 
pathway. The high glycolytic rate during the Warburg effect thereby meets the requirement for the production of nucleotides and amino acids for the creation of new biological building blocks. In addition, fatty acids needed for membrane lipid synthesis are synthesized from citrate in the cytosol through the action of ATP-citrate lyase (ACL) to generate acetyl-CoA. This process requires NADPH reducing equivalents, which can be generated through several steps within the pentose phosphate pathway. Furthermore, the glycolytic intermediate dihydroxyacetone phosphate (DHAP) is a source of the glycerol that combines with fatty acids to form triacylglycerols. Precursors of serine, glycine, and methyl groups are also derived from intermediates of glycolysis. The increase in uptake of glucose combined with highly active glycolysis result in an improved availability of these precursors, meeting all the requirements of increased biomass during cell proliferation.

One key mediator of the Warburg effect is the transcription factor Hypoxiainducible factor- $1 \alpha$ (Hif- $1 \alpha)$. The Hif transcription factors were first identified as proteins that respond to decreases in available oxygen or hypoxia. They are highly conserved across species and form transcriptional heterodimeric complexes composed of an $\alpha$ and a $\beta$ subunit. Several studies have implicated a role for HIF-1 $\alpha$ as a mediator of the Warburg effect [6-9]. Recent findings suggest a link between Hif- $1 \alpha$ and aggressive cancers, where elevated Hif- $1 \alpha$ activity is shown to mediate the Warburg effect in clear cell renal carcinoma, as well as occurring in the hereditary von Hippel-Landau (VHL) cancer syndrome $[10,11]$. The role of Hif- $1 \alpha$ is in the induction of enzymes in glycolysis, notably Hexokinase 2, glucose-6-phosphate isomerase, triosephosphate isomerase and pyruvate kinase M2 (PKM2) [12]. As discussed below however, Hif-1 $\alpha$ can also be activated under normoxia by succinate with Hif- $1 \alpha$ therefore still playing a role in tumors that are well vascularized.

The Warburg effect in inflammation and innate immunity

Immune cells, after becoming activated, also have the ability to switch from oxidative phosphorylation to aerobic glycolysis in a similar manner to a tumor cell $[13,14]$. This metabolic conversion suggests a potential novel target for controlling excessive inflammation and improper immune responses.

In response to invading pathogens the innate immune system is the first line of defense. Detection of these pathogens is made possible by the use of pattern recognition receptors (PRRs), that recognize pathogen-associated molecular patterns (PAMPs), as well as damage-associated molecular patterns (DAMPs). PRRs can be membrane bound such as the Toll-like receptors (TLRs) and C-type lectin receptors (CLR) or they can be cytosolic such as the RIG-I and the NOD-like receptors (NLRs) which include NODs and NLRPs. Once triggered by a bound ligand the receptors elicit a cascade of events resulting in the activation of transcription factors such as NFKB; and give rise to the production of a wide 
array of pro-inflammatory cytokines. A large part of pro-inflammatory innate immune response culminates in an increased expression of two prototypic proinflammatory cytokines, interleukin-1 $\beta$ (IL-1 $\beta$ ) and tumor necrosis factor- $\alpha$ (TNF $\alpha$ ). Both IL-1 $\beta$ and TNF $\alpha$ have been implicated as main effectors of both autoimmune diseases and metabolic disorders [15].

Naïve immune cells including neutrophils, dendritic cells (DCs), and macrophages, when activated using TLR ligands or pro-inflammatory cytokines, switch their metabolism from oxidative phosphorylation to aerobic glycolysis. Krawczyk et al. [14] describe this phenomenon in DCs and also demonstrate how TLR stimulation is essential for dendritic cell maturation. Stimulating DCs with ligands for TLR4, TLR2, and TLR9 causes an increase in glycolytic rate and glucose consumption, promoting expression of glucose transporter 1 (GLUT1) mRNA as well as protein, and increasing lactate production. The Akt/phosphatidylinositide 3-kinase (PI3K) pathway promotes this metabolic switch and anti-inflammatory signals such as $5^{\prime}$ AMP-activated protein kinase (AMPK) activation as well as interleukin-10 (IL-10) have an inhibitory role.

Macrophages also have the ability to alter their metabolism. Activating macrophages using interferon- $\gamma$, or certain ligands for TLRs can cause these cells to take on the "classic" M1 phenotype, which will clear bacteria by expressing pro-inflammatory cytokines and reactive oxygen species (ROS). The contrasting "alternative" M2 macrophage phenotype develops after the cells are stimulated using other cytokines such as IL-4 and IL-13, or TGF $\beta$. Differentiation of a macrophage into the M1, but not the M2, phenotype also involves a switch to aerobic glycolysis. One theory explaining the necessity to generate ATP in this manner has been presented by West et al. [16] who demonstrate the importance of mitochondrial ROS in bacterial clearing. Activation of TLR2, 4, and 6 results in mitochondria migrating to the phagosome. The signaling adaptor, TNF receptorassociated factor 6 (TRAF6), subsequently translocates to the mitochondria. Here it interacts with evolutionary conserved signaling intermediate in Toll pathway (ECSIT), leading to an increased production of mitochondrial ROS. The latter combines with the cytoplasmic ROS resulting from phagosomal NADPHoxidase dependent respiratory burst, to aid bacterial killing. Since mitochondrial ROS production requires complex I of the respiratory chain, increased glycolysis will compensate for the switch in mitochondrial function away from ATP production towards ROS production. Glycolysis thereby becomes an important source of ATP, both for energy, and also for the maintenance of the mitochondrial membrane potential, which will fall, because of the decrease in the TCA cycle and decreased electron transport.

The Warburg effect beyond glycolysis: Alterations in the TCA cycle

Although Warburg predominantly focused on glycolysis, suggesting an attenuation in mitochondrial metabolism, he was somewhat incorrect [2]. 
Although mitochondrial respiration is repressed in tumor cells, it now turns out that certain TCA cycle intermediates are elevated and have important functions, notable examples being citrate which is generated by citrate synthase and succinate generated by the enzyme succinyl-CoA synthetase. In effect, the TCA cycle becomes anabolic as opposed to catabolic, citrate being withdrawn for lipid biosynthesis.

\section{Citrate in LPS-activated macrophages}

An elevation in citrate also occurs in lipopolysaccharide (LPS)-activated macrophages. The citrate enters the cytosol aided by the mitochondrial citrate carrier (CIC), which in return transports cytosolic malate to the mitochondria $[17,18]$. Cytosolic citrate is then cleaved by citrate lyase into acetyl-CoA and oxaloacetate. Oxaloacetate produces NADPH, an important cofactor in the generation of nitric oxide (NO)(L-arginine converted into citrullin), and ROS (by NADPH-oxidase) whereas acetyl-CoA can be directly used for fatty acid biosynthesis, providing a precursor to prostaglandin synthesis. Since inhibition of CIC leads to a significant reduction in production of LPS-induced NO, prostaglandins, and ROS production, Infantino and colleagues [17] speculate that the citrate exported from the mitochondria may, via acetyl-CoA, play a key role in LPS signaling (Fig. 1).

Succinate as a signal from the TCA cycle

Succinate levels are also elevated in certain tumors, where it inhibits prolyl hydroxylases (PHDs). These enzymes actually generate succinate from $\alpha$ ketoglutarate, acting as dioxygenases. Succinate elevation from the TCA cycle has a negative feedback effect on PHDs, hence stabilizing Hif-1 $\alpha$ [19]. Succinate elevation is also a feature of LPS-activated macrophages (Fig. 1). These cells also have a highly glycolytic profile [20]. The source of this succinate is a combination of two processes. Firstly glutamine-dependent anaplerosis generates succinate, whereby glutamine is used to produce glutamate and subsequently $\alpha$ ketoglutarate during an anaplerotic reaction, or glutaminolysis. Secondly succinate is also generated, again using glutamine via glutamate, through the $\gamma$ aminobutyric acid (GABA)-shunt. This involves transamination of $\alpha$ ketoglutarate from the TCA by GABA $\alpha$-oxoglutarate transaminase generating Lglutamic acid. This step of the GABA shunt can be irreversibly inhibited by Vigabatrin (Fig. 2). Glutamic acid decarboxylase (GAD) then catalyzes the decarboxylation of glutamic acid to form GABA. GABA, once converted to succinic semialdehyde, becomes a source of succinate. The increase in succinate facilitates the signal leading to stabilization of Hif- $1 \alpha$, which in turn positively regulates the pro-inflammatory cytokine IL-1 $\beta$ and other Hif-dependent genes (Fig. 1). In addition to this, Hif- $1 \alpha$ itself down-regulates mitochondrial oxidative phosphorylation by inducing pyruvate dehydrogenase kinase 1 (PDK1) and 3 
(PDK3), both of which in turn also inactivate PHD, allowing for further stabilization of Hif- $1 \alpha$ [21-23].

Figure 2.

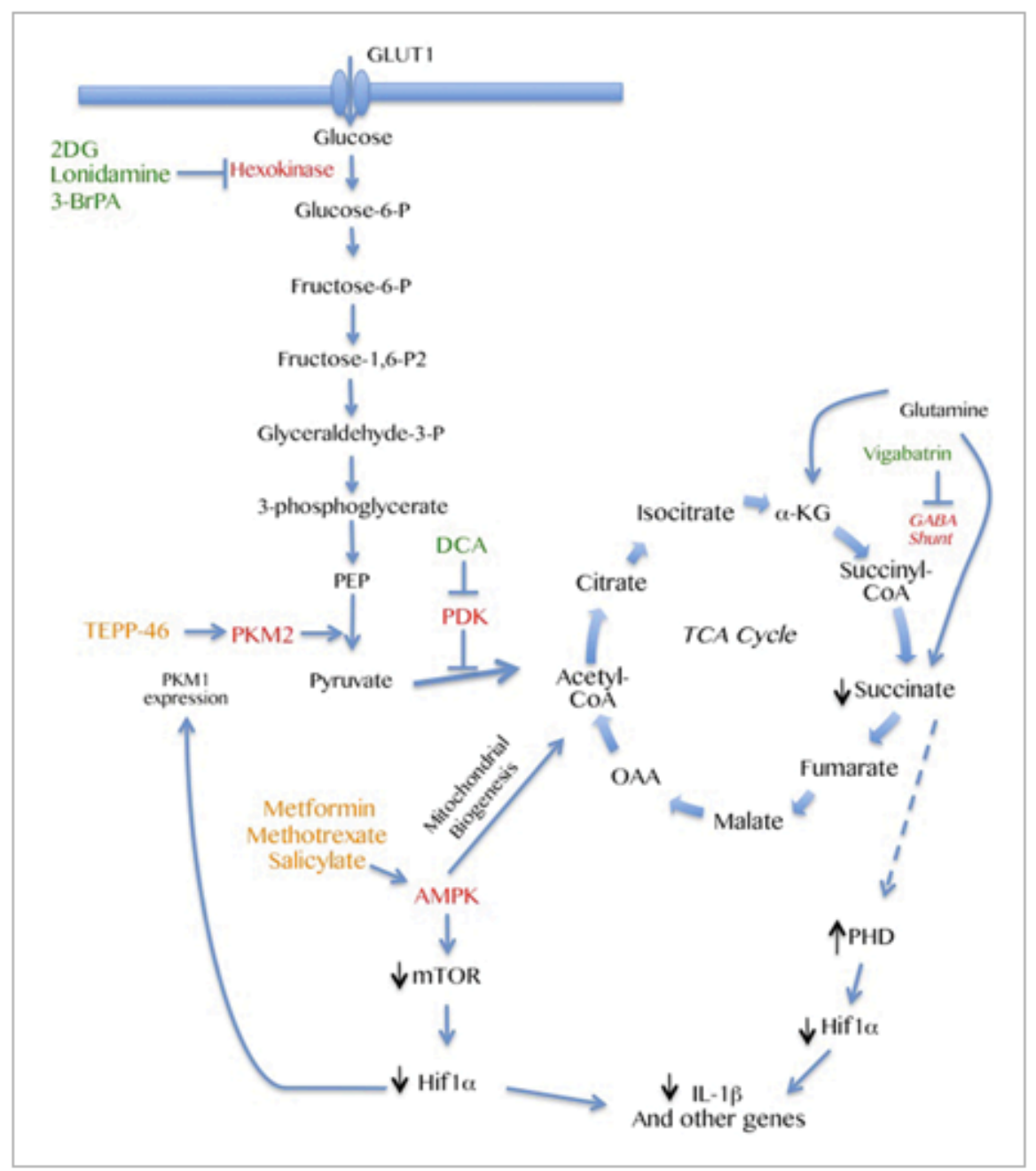

Potential targets in metabolism for anti-inflammatory therapeutics. Targets are depicted in red, inhibitory compounds are indicated in green, and activating compounds are shown in orange. Turning off the Warburg effect by promoting oxidative phosphorylation, and down regulating glycolysis in activated immune cells may provide us with a novel therapeutic strategy when treating inflammatory diseases. One important target in glycolysis is Hexokinase which can be inhibited using 2DG, Lonidamine and 3-BrPA. Activation of PKM2 by small molecules such as TEPP-46 may promote an increased flux of pyruvate into the mitochondria where it enters the TCA cycle at the point of acetyl-CoA. Inhibiting PDK using a compound such as DCA will, through activation of PDH, also allow for increased mitochondrial respiration. Increased succinate levels may be inhibited using the GABA shunt inhibitor Vigabatrin. Since increased succinate has been shown to enhance Hif- $1 \alpha$ and IL-1 $\beta$ during inflammation, Vigabatrin 
mediated decrease in succinate leads to decreased Hif- $1 \alpha$ stability, and a decrease in expression of the pro-inflammatory cytokine IL-1 $\beta$ [20]. Targeting and activating AMPK using metformin, methotrexate, or salicylate will lead to increased mitochondrial biogenesis, decreased mTOR activity, and again decreased Hif-1 $\alpha$. Furthermore, since Hif- $1 \alpha$ promotes expression of PKM2, a decrease in Hif- $1 \alpha$ may result in an increase PKM1 expression, promoting oxidative phosphorylation.

Another consequence of elevated intracellular succinate is an increase in protein lysine-succinylation, a recently described posttranslational modification [24]. Lysine-succinlyation of histones has been described as well as succinylation of mitochondrial metabolic enzymes [25]. In addition, LPS causes a twofold increase in lysine-succinylation of total cellular protein [20]. Several members of the sirtuin family (Sirt1-7 in mammals) have well studied NAD-dependent deacetylase activity. However, a recent study by Du et al. revealed Sirt5 to have a strong lysine desuccinylase and demalonylase activity. This would allow for regulation of the consequences of lysine-succinylation [25]. Sirt5 expression is inhibited by LPS [20], resulting in reduced desuccinylase activity, which contributes to the LPS-induced increase in protein lysine-succinylation observed.

Succinate also appears to act as an endogenous danger signal released by mitochondria in response to infection. It can signal through GPR91, which synergizes with TLRs [26]. It has also been linked to diseases such as peritonitis, and can be detected in urine and plasma in rodent models of hypertension and metabolic disease $[27,28]$. The elevation in succinate will therefore have multiple consequences: activation of Hif- $1 \alpha$, protein succinylation, and activation of the pro-inflammatory receptor GPR91.

The Warburg effect and adaptive immunity

As well as having a role in the activation of innate immune cells such as macrophages, evidence is also emerging that the Warburg effect is of key importance in adaptive immunity. The complex network of specialized T cell populations required for the adaptive immune system to appropriately respond to infection are essentially derived from a common pool of precursor naïve CD4+ $\mathrm{T}$ cells. Following T cell receptor (TCR) activation these naïve CD4 T cells will then differentiate, depending on extracellular cytokine and environmental signals, into one of several lineages of T helper (Th) cells. These include Th17 cells, and regulatory T cells (Tregs). Th17 cells produce the pro-inflammatory cytokine interleukin-17 (IL-17) and although important in infection, Th17 cells play an instrumental role in the development of various autoimmune diseases such as rheumatoid arthritis (RA), psoriasis, autoimmune uveitis, juvenile diabetes, and multiple sclerosis (reviewed in [29]). Tregs on the other hand predominantly display anti-inflammatory properties, and failing adequate Treg 
responses will for example lead to a dysregulation in the inflammatory process [30-32].

An interesting comparison can be made when looking at the metabolic processes in the pro-inflammatory "M1" macrophage and inflammatory T cells such as the Th17 which both display an increased glycolytic metabolism. Equally, the antiinflammatory "M2" macrophage which has a high oxidative phosphorylation rate has a similar metabolism to the anti-inflammatory counterpart Treg of the adaptive immune system [33]. Dang et al. demonstrate how Hif- $1 \alpha$ promotes differentiation of Th17 cells through direct transcriptional activation of ROR $\gamma$ t, the key transcription factor for Th17 cells. This allows for interaction and recruitment of histone acetyl transferase p300 to the promoter of IL-17. The authors also show how Hif- $1 \alpha$ is able to bind Foxp3, the master regulator of Tregs. This targets Foxp3 for degradation, and in doing so attenuates Treg development [34]. Furthermore, mice with Hif1 $\alpha$ deficient T cells failed to generate Th17 cells, but displayed an increased Treg population. These mice were also resistant to developing Th17-dependent experimental autoimmune encephalitis, a model of multiple sclerosis. Importantly, inhibiting glycolysis promotes Treg cell differentiation, and reciprocally reduces Th17 cell differentiation [12]. This again points to the Warburg effect, and glycolysis as important in a pro-inflammatory cell.

Targeting the Warburg effect in inflammation

Since the Warburg effect occurs in all cancers, and since it is critical for tumor progression, developing an approach targeting it therapeutically may resolve the malignancy or halt progression of many different types of cancers [35, 36]. Since, as discussed above, Warburg metabolism is also relevant to inflammation, some of these approaches of disrupting it may also be applicable to the field of inflammatory disorders. Inhibition of glycolysis or re-engaging the mitochondrial oxidative respiration of an overactive "M1" macrophage, DC, or Th17 may result in a dampening effect on inflammation. Potential targets are shown in Figure 2.

Hexokinase as a target

Reactions of glycolysis are catalyzed by numerous enzymes and enzymatic complexes, and some of these may represent potential therapeutic targets. One important target is hexokinase, on account of its important roles in both glycolysis and apoptosis. 2-Deoxyglucose (2DG), which is in clinical trials for a range of cancers [36], is a glucose analogue that acts as a potent competitive inhibitor of glycolysis at the level of hexokinase. We have found that 2DG can also inhibit the production of IL-1 $\beta$ by LPS by somehow decreasing succinate levels, probably by promoting mitochondrial metabolism [20]. This might occur 
as a response to decreased glycolysis, the cell responding by boosting mitochondrial biogenesis, which would include an increase in succinate dehydrogenase. 2DG, or adducts thereof that more specifically inhibit hexokinase, might therefore be of use in treating inflammatory disorders (Fig. 2).

PKM2 as a target

Since PKM2 is an important regulator of the Warburg effect in cancer (reviewed in [37]), it may prove an auspicious target for interfering with glycolysis and consequences of anabolic metabolism both in inflammation and cancer.

A resting cell predominately expresses PKM1, which through its high enzymatic activity will promote pyruvate entering the TCA cycle allowing for oxidative respiration to occur (Fig. 1). However PKM2 is the major isoform expressed in cancer cells [38-40]. It has been shown to be required for tumor growth and necessary for the Warburg effect. PKM2 has a far lower enzymatic activity than the alternatively spliced PKM1. PKM2 will promote rapid glycolytic energy production, and allow for intermediates of glycolysis to accumulate and enter alternative pathways to generate amino acids, nucleic acids, and fatty acids without a build up of reactive oxygen species. Moreover, the phoshoenolpyruvate (PEP) to pyruvate catalytic activity of PKM2 is regulated by the formation of a catalytically active tetramer or a less enzymatically active dimer, the latter of these providing a growth advantage for tumor progression. An alternative glycolytic pathway has been identified whereby cells expressing the less enzymatically active PKM2 may still produce pyruvate. This occurs by means of PEP dependent phosphorylation of phosphoglycerate mutase (PGAM1) which will lead to pyruvate generation in the absence of active pyruvate kinase [41].

PKM2 expression was recently shown to be regulated by activation of the mTOR signaling pathway (Fig. 3). The mechanism by which this occurs involves mTOR positively regulating the transcriptional activity of Hif- $1 \alpha$, which through c-mycheterogeneous nuclear ribonucleoproteins (hnRNPs) can then preferentially promote gene splicing resulting in the PKM2 isoform. This points to mTOR as another important regulator of the Warburg effect [42].

Figure 3. 


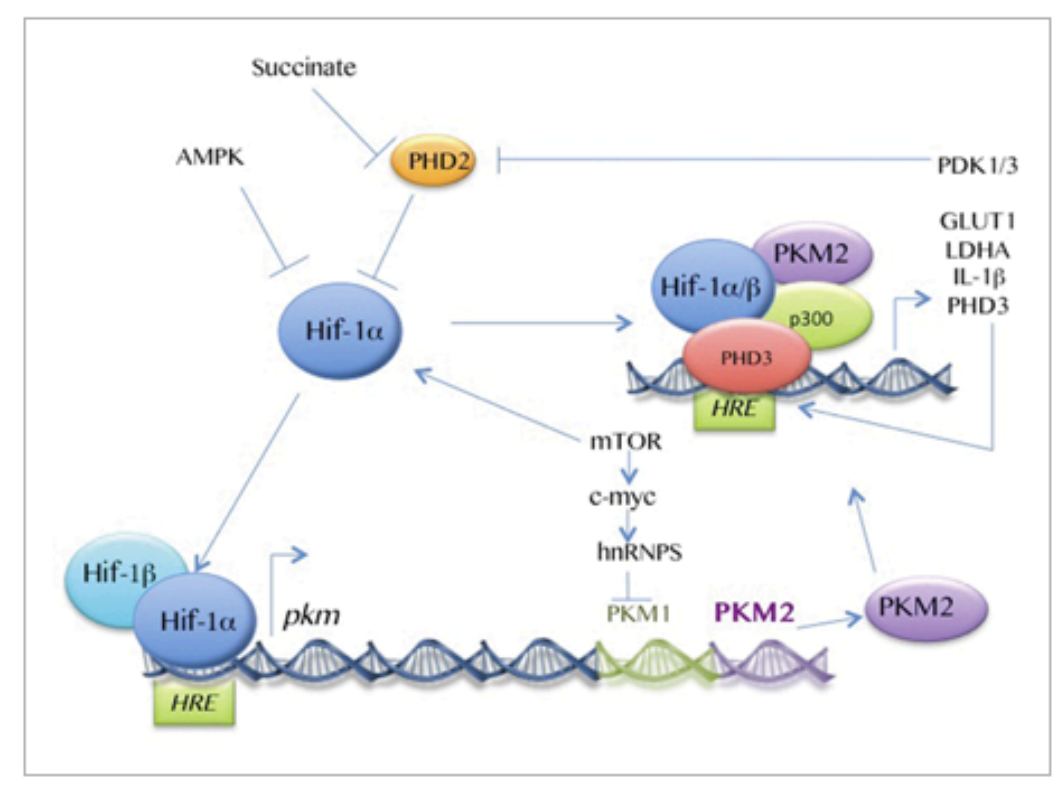

Simplified diagram depicting the role of Hif- $1 \alpha$ in the regulation of glycolysis. Hif$1 \alpha$ plays a central role in multiple functions within the cell, including cell survival, angiogenesis, cell migration, transport, growth, and apoptosis. In addition to this Hif- $1 \alpha$ positively regulates glycolysis on several levels. PKM1 and PKM2 are the alternatively spliced products of the pkm primary RNA transcript with PKM1 and PKM2 mRNA containing sequences encoded by exon 9 or exon 10, respectively. By binding to the hypoxia response element (HRE) of the pkm gene, the Hif- $1 \alpha$ and Hif- $1 \beta$ heterodimer complex positively promotes expression of PKM2 [73]. Preferential splicing of pkm occurs through activation of heterogenous nuclear ribonucleoproteins (hnRNPs) I, AI, and A2, which bind to RNA sequences encoded by exon 9 thereby inhibiting PKM1-specific mRNA splicing. Transcription of hnRNPs are in turn regulated by c-myc. Mammalian target of rapamycin (mTOR) promotes expression of PKM2 by activating c-myc and Hif- $1 \alpha$. Resulting PKM2 can combine with Hif- $1 \alpha$, Hif- $1 \beta$, p300, and PHD3 to promote expression of pro-glycolytic components as well as pro-inflammatory cytokines such as IL-1 $\beta$.

PKM2 has also been shown to form a nuclear complex with Hif- $1 \alpha$ and prolyl hydroxylase domain enzyme 3 (PHD3) following which PKM2 can positively regulate expression of Hif- $1 \alpha$ target genes including key metabolic players such as GLUT1, PHD3, PDK1, LDHA, and PKM2, thereby further positively feeding a highly glycolytic metabolic profile [43, 44] (Fig. 3). A dual kinase role for PKM2 has been suggested, functioning either as a pyruvate kinase, or as more recently observed, as a protein kinase. Gao et al. $[45,46]$ demonstrate the opposing effects growth signals may have, regulating these two enzymatic activities by controlling the dimer or tetramer formation of PKM2.

PKM2 as a dimer acts in a pro-glycolytic manner, both, as a result of low enzymatic activity, which promotes lactate fermentation, but also through the 
ability of a dimer to translocate into the nucleus where it positively regulates Hif$1 \alpha$ responsive genes [47] (Fig. 3). Anastasiou et al. [48] show that small molecular activators of PKM2 can mimic the naturally occurring PKM2 activator fructose-1,6-bisphosphate (FBP). PKM2 will then enter a tetrameric formation, which will thereby activate the catalytic function of PKM2. The tetrameric formation of PKM2 may also prevent PKM2 from entering the nucleus, thereby having a double negative effect on glycolysis by down regulating Hif- $1 \alpha$ responsive genes such as GLUT1. Since PKM2 has long been earmarked as a potential target for cancer therapy, results presented by Anastasiou et al. look particularly interesting. In addition, studies in mice demonstrate that one of these activators, TEPP-46, when given orally achieved activation of PKM2, and tetramer formation in vivo, which correlated with xenograft tumors displaying impaired growth and a lower concentration of lactate, ribose phosphate and serine [48]. It remains to be determined whether this approach will prove effective in models of inflammation. Figure 1 depicts the potential roles of PKM1 and PKM2 in the anti-inflammatory and pro-inflammatory cell phenotypes, with the targeting of PKM2 shown in Figure 2.

PDK as a target

Another potential target lies with mitochondrial pyruvate dehydrogenase kinase (PDK) (reviewed in [49]). As an inhibitor of PDH, it regulates the uptake of cytosolic pyruvate into the TCA cycle at the point of acetyl-CoA. PDK was shown over 20 years ago to be activated in sepsis, leading to reduced mitochondrial PDH activity in septic animals [50]. Inhibiting PDK using either small interfering RNA (siRNA) or dichloroacetate (DCA) would allow for an increased mitochondrial respiration and a reduction in aerobic glycolysis (Fig. 2). Additional benefits to this approach, such as that of activating PKM2, include decreased production of mitochondrial ROS, and inhibition of Hif- $1 \alpha$. DCA, a small 150 Da molecule that easily penetrates the cell membrane, specifically inhibits PDK2, allowing for activation of PDH. Apart from showing great promise in pre-clinical trials for a vast range of cancers [51-55], DCA has also been successfully tested in clinical trials of congenital mitochondrial disorders in order to limit lactic acidosis [56].

AMPK as a target

Another important regulator of oxidative phosphorylation is the metabolic master switch AMPK. Made up of three subunits $\alpha, \beta$, and $\gamma$, each subunit existing in up to three different isoforms, it forms a heterotrimeric protein that senses shifts in cellular AMP:ATP levels. Once activated by metabolic stress, or other triggers of an increased intracellular AMP/ATP or ADP/ATP ratio, mammalian AMPK promotes mitochondrial biogenesis and an increased cellular uptake of glucose through an increased expression of GLUT4 [57]. Furthermore AMPK promotes the $\beta$-oxidation of fatty acids [58] and increased expression of a number of enzymes critical for oxidative phosphorylation [59]. Since the activation of AMPK results in actions aimed at restoring energy homeostasis of 
the cell, through activation of catabolic pathways to generate ATP, drugs activating AMPK will not only be anti-inflammatory, but may also act in an "antiWarburg" manner inhibiting cancer growth (reviewed in $[33,60]$ ).

AMPK has been shown to regulate a key inflammatory protein called NLRP3. This protein occurs in multiprotein complexes called inflammasomes. Inflammasomes are so called since they also contain the enzyme Caspase-1, which processes the pro-inflammatory cytokine IL-1 $\beta$ to its mature active form. NLRP3 inflammasome activation and subsequent elevated IL-1 $\beta$ expression are a central feature of several metabolic disorders including gout, atherosclerosis, and obesity.

The proposed mechanism of NLRP3 activation during aerobic glycolysis involves down-regulation of AMPK activity. AMPK promotes a selective form of autophagy called mitophagy involving autolysosomal degradation of mitochondria. Decreased AMPK activity leads to down-regulation of mitophagy, allowing for a build up of mitochondrial ROS, a potent activator of NLRP3 [33, 61, 62]. Furthermore, NLRP3 inflammasome activation is elevated in myeloid cells from type 2 diabetic patients and treatment using metformin, an anti-diabetic drug that activates AMPK, inhibits the maturation of IL-1 $\beta$ in macrophages derived from these patients, further supporting a role for AMPK in NLRP3 activation [63] (Fig. 2).

Other drugs such as methotrexate and salicylate (the major breakdown product of aspirin) also activate AMPK (Fig. 2). AMPK has been shown to be antiinflammatory, acting through multiple mechanisms, with induction of mitochondrial biogenesis only contributing in part to a wide rage of effects. Both salicylate and metformin have demonstrated promising anti-cancer properties [64]. Metformin has been widely used in treating type II diabetes for over 40 years, where it in part acts by suppressing hepatic glucose production through AMPK. However, metformin also confers benefits that include a reduction in the risk of myocardial infarction, stroke, lower cancer mortality, as well as attenuating airway inflammation [65-68]. A recent study by Nath et al. [69] also demonstrates a dramatic inhibitory effect of metformin on the disease progression of a murine model of multiple sclerosis, experimental autoimmune encephalomyelitis (EAE). The concept of AMPK activation being antiinflammatory through inducing a state of pseudo-starvation is reviewed in [33].

AMPK is known to inhibit the mTOR complex (mTORC1) pathway. It does this through two possible routes: phosphorylation and inactivation of Raptor; phosphorylation and activation of the Rheb GTPase-activating protein TSC2. Since the PI3K/Akt/mTOR pathway is critical for upregulation of PKM2 expression and subsequent aerobic glycolysis [42], activation of AMPK may be 
anti-inflammatory, because of an inhibitory effect on the mTOR pathway. This could limit Hif- $1 \alpha$ activation [70], or as described above limit PKM2 expression (Fig. 2). However, direct inhibitors of mTOR may also prove effective in targeting glycolysis. Examples of such inhibitors showing promising outcomes in clinical trials include perifosine, an inhibitor of Akt [71], and the mTORC1 inhibitor Temsirolimus, which both show activity in advanced renal cell carcinoma [36, 72].

\section{Conclusion}

Tumor cells have a fundamentally different metabolic profile from that of normal differentiated tissue. This shift towards a high rate of glycolysis, or Warburg effect, has long been recognized as a hallmark of cancer. However, the Warburg effect is steadily becoming established as an important phenomenon in inflammatory immune responses. Upon activation of pro-inflammatory immune cells, a high rate of glycolysis may provide the means to meet an increased demand for biosynthetic precursors used for synthesis of pro-inflammatory proteins.

The accumulated knowledge of pathways and regulatory enzymes involved in the Warburg effect is challenging because of the complexities involved. Nonetheless, the insights being made are providing us with numerous potential drug targets for treating inflammatory diseases and metabolic disorders, as well as cancer. Without doubt, there is already evidence that inhibitors of the Warburg effect have an overlapping effect when treating inflammation and cancer, as in the case of metformin. Our continuously improving understanding of metabolism in inflammatory disorders, cancer, and metabolic disease provide us with valuable insight and potential novel therapeutic approaches. 


\section{References}

1. Warburg 0. 1923. Metabolism of tumours. Biochem Z 142: $317-33$.

2. Koppenol WH, Bounds PL, Dang CV. 2011. Otto Warturg's contributions to current concepts of cancer metabolism. Nat Rev Cancer 11: 325-37.
3. Warburg 0 . 1925. Iron, the oxygen-carrier of respiration-ferment. Science 61: 575-82.

4. Racker E. 1972. Bioenergetics and the problem of tumor growth. Am So 60: $56-63$.

5. Turner JS, Brittain EG. 1962. Oxygen as a factor in photosynthesis. Bio Rev Camb Philos Soc 37: 130-70.

6. Larbi A, Zelba H, Goldeck D, Pawelec G. 2010. Induction of HIF-1alpha and the glycolytic pathway alters apoptotic and differentiation profiles of activated human T cells. J Leukoc Bial 87: 265-73.

7. Denko NC. 2008. Hypoxia, HIF1 and glucose metabolism in the solid tumour. Nat Rev Cancer 8: 705-13.

8. Maynard MA, Ohh M. 2007. The role of hypoxia-inducible factors in cancer. Cell Mol Life Sci 64: 2170-80.

9. Darekar S, Georgiou K, Yurchenko M, Yenamandra SP, et al. 2012. Epstein-Barr virus immortalization of human B-cells leads to stabilization of hypoxia-induced factor 1 alpha, congruent with the Warburg effect. PLoS One 7: 042072 .

10. Kondo K, Klco J, Nakamura E, Lechpammer M, et al. 2002. Inhibition of HIF is necessary for tumor suppression by the von Hippel-Lindau protein. Cancer Cell 1: $237-46$.

11. Semenza GL. 2010. Defining the role of hypoxia-inducible factor 1 in cancer biology and therapeutics. Oncogene 29: 625-34.

12. Shi LZ, Wang R, Huang G, Vogel P, et al. 2011. HIF1alpha-dependent glycolytic pathway orchestrates a metabolic checkpoint for the differentiation of TH17 and Treg cells. J Exp Med 208: 1367-76.

13. Garedew A, Henderson SO, Moncada S. 2010. Activated macrophages utilize glycolytic ATP to maintain mitochondrial membrane potential and prevent apoptotic cell death. Celli Death Differ 17: 1540-50.

14. Krawczyk CM, Holowka T, Sun J, Blagih J, et al. 2010. Toll-like receptor-induced changes in glycolytic metabolism regulate dendritic cell receptor-induced changes in g.

15. Dinarello CA, Simon A, van der Meer JW. 2012. Treating inflammation by blocking interleukin-1 in a broad spectrum of diseases. Nat Rev Drug by blocking interleu

16. West AP, Brodsky IE, Rahner C, Woo DK, et al. 2011. TLR signalling augments macrophage bactericidal activity through mitochondrial ROS.

17. Infantino V, Convertini P, Cucci L, Panaro MA, et al. 2011. The mitochondrial citrate carrier: a new player in inflammation. Biochem $J$ 438: $433-6$

18. O'Neill LA. 2011. A critical role for citrate metabolism in LPS signalling. Biochem J 438: e5-6.

19. Selak MA, Armour SM, MacKenzie ED, Boulahbel H, et al. 2005. Succinate links TCA cycle dysfunction to oncogenesis by inhibiting HIF-alpha prolyl hydroxylase. Cancer Cell 7: 77-85.

20. Tannahill GM, Curtis AM, Adamik J, Palsson-McDermott EM, et al. 2013. Succinate is an inflammatory signal that induces IL-1beta through HIF-1alpha. Nature 496: 238-42.

21. Kim JW, Tchernyshyov I, Semenza GL, Dang CV. 2006. HF-1mediated expression of pyruvate dehydrogenase kinase: a metabolic switch required for cellular adaptation to hypoxia. Cell Metabo/ 3: 177-85.

22. Lu CW, Lin SC, Chen KF, Lai YY, et al. 2008. Induction of pyruvate dehydrogenase kinase- 3 by hypoxia-inducible factor -1 promotes metabolic switch and drug resistance. J Biol Chem 283: 28106-14.

23. Papandreou I, Cairns RA, Fontana L, Lim AL, et al. 2006. HIF-1 mediates adaptation to hypoxia by actively downregulating mitochondria oxygen consumption. Cell Metabol 3: 187-97.

24. Zhang Z, Tan M, Xie Z, Dai L, et al. 2011. Identification of lysine succinylation as a new post-translational modification. Nat Chem Biol 7 $58-63$.

25. Du J, Zhou Y, Su X, Yu JJ, et al. 2011. Sirt5 is a NAD-dependent protein lysine demalonylase and desuccinylase. Science 334: 806-9.

26. Rubic T, Lametschwandtner G, Jost S, Hinteregger S, et al. 2008. Rubic T, Lametschwandtner G, Jost S, Hinteregger S, et al. 2008.
Triggering the succinate receptor GPR91 on dendritic cells enhances Triggering the succinate receptor
immunity. Nat Immuna/ 9: 1261-9.

27. Sadagopan N, Li W, Roberds SL, Major T, et al. 2007. Circulating succinate is elevated in rodent models of hypertension and metabolic disease. Am J Hypertens 20: 1209-15.

28. Rotstein OD, Pruett TL, Fiegel VD, Nelson RD, et al. 1985. Succinic acid, a metabolic by-product of Bacteroides species, inhibits polymorphonuclear leukocyte function. Infect immun 48: 402-8.

29. Kurebayashi Y, Nagai S, Ikejiri A, Koyasu S. 2013. Recent advances in understanding the molecular mechanisms of the development and function of Th17 cells. Genes Cells 18: 247-65.

30. Mayne CG, Williams CB. 2013. Induced and natural regulatory T cells in the development of inflammatory bowel disease. Inflamm Bowel Dis
31. Ishikawa D, Okazawa A, Corridoni D, Jia LG, et al. 2013. Tregs are dysfunctional in vivo in a spontaneous murine model of Crohn's disease. dysfunctional in vivo in a spo

32. Miyara M, Sakaguchi S. 2011. Human FoxP $3(+) C D 4(+)$ regulatory $T$ cells: their knowns and unknowns. Immunol Cell Biol 89: 346-51.

33. O'Neill LA, Hardie DG. 2013. Metabolism of inflammation limited by AMPK and pseudo-starvation. Nature 493: 346-55.

4. Dang EV, Barbi J, Yang HY, Jinasena D, et al. 2011. Control of $\mathrm{T}(\mathrm{H}) 17 /$ T(reg) balance by hypoxia-inducible factor 1 . Cell 146: 772-84.

35. Vamecq J, Colet JM, Vanden Eynde JJ, Briand G, et al. 2012. PPARs: interference with Warburg' effect and clinical anticancer trials. PPAR Res 2012: 304760 .

36. Zhao Y, Liu H, Riker Al, Fodstad O, et al. 2011. Emerging metabolic targets in cancer therapy. Front Biosci 16: 1844-60.

37. Jiang L, Deberardinis RJ. 2012. Cancer metabolism: when more is less. Nature 489: 511-2.

38. Christofk HR, Vander Heiden MG, Harris MH, Ramanathan A, et al. 2008. The M2 splice isoform of pyruvate kinase is important for cancer metabolism and tumour growth. Nature 452: 230-3.

39. Cairns RA, Harris IS, Mak TW. 2011. Regulation of cancer cell metabolism. Nat Rev Cancer 11: 85-95.

40. Tamada M, Suematsu M, Saya H. 2012. Pyruvate kinase M2: multiple faces for conferring benefits on cancer cells. Clin Cancer Res 18: 5554-61.

41. Vander Heiden MG, Locasale JW, Swanson KD, Sharfi H, et al. 2010. Evidence for an alternative glycolytic pathway in rapidly proliferating cells. Science 329: 1492-9.

42. Sun Q, Chen X, Ma J, Peng H, et al. 2011. Mammalian target of rapamycin up-regulation of pyruvate kinase isoenzyme type M2 is critical for aerobic glycolysis and tumor growth. Proc Nat' Acad Sci USA 108: for aerobic

43. Yang W, Zheng Y, Xia Y, Ji H, et al. 2012. ERK1/2-dependent Yang W, Zheng Y, Xia Y, Ji H, et al. 2012. ERK1/2-dependent
phosphorylation and nuclear translocation of PKM2 promotes the Warburg effect. Nat Cell Biol 14: 1295-304.

44. Pouyssegur J, Dayan F, Mazure NM. 2006. Hypoxia signalling in cancer and approaches to enforce tumour regression. Nature 441:

5. Gao X, Wang H, Yang JJ, Chen J, et al. 2013. Reciprocal regulation of protein kinase and pyruvate kinase activities of pyruvate kinase M2 by growth signals. J Bial Chem 288: 15971-9.

46. Gao X, Wang H, Yang JJ, Liu X, et al. 2012. Pyruvate kinase M2 regulates gene transcription by acting as a protein kinase. Mol Cell 45: 598-609.

47. Luo W, Hu H, Chang R, Zhong J, et al. 2011. Pyruvate kinase M2 is a PHD3-stimulated coactivator for hypoxia-inducible factor 1. Cell 145: $732-44$.

48. Anastasiou D, Yu Y, Israelsen WJ, Jiang JK, et al. 2012. Pyruvate kinase M2 activators promote tetramer formation and suppress tumorigenesis. Nat Chem Bial 8: 839-47.

49. Sutendra G, Michelakis ED. 2013. Pyruvate dehydrogenase kinase as a novel therapeutic target in oncology. Front Oncol 3: 38.

50. Vary TC. 1991. Increased pyruvate dehydrogenase kinase activity in response to sepsis. Am J Physiol 260: E669-74.

51. Sanchez-Arago M, Chamorro M, Cuezva JM. 2010. Selection of cancer cells with repressed mitochondria triggers colon cancer progression. Carcinogenesis 31: 567-76.

52. Cao W, Yacoub S, Shiverick KT, Namiki K, et al. 2008. Dichloroacetate (DCA) sensitizes both wild-type and over expressing Bcl-2 prostate cancer cells in vitro to radiation. Prostate 68: 1223-31.

53. Wong JY, Huggins GS, Debidda M, Munshi NC, et al. 2008. Dichloroacetate induces apoptosis in endometrial cancer cells. Gynecol Oncal 109: $394-402$.

54. Sun RC, Fadia M, Dahlstrom JE, Parish CR, et al. 2010. Reversal of the glycolytic phenotype by dichloroacetate inhibits metastatic breast cancer cell growth in vitro and in vivo. Breast Cancer Res Treat 120: $253-60$

55. Michelakis ED, McMurtry MS, Wu XC, Dyck JR, et al. 2002. Dichloroacetate, a metabolic modulator, prevents and reverses chronic hypoxic pulmonary hypertension in rats: role of increased expression and activity of voltage-gated potassium channels. Circulation 105: $244-50$

6. Stacpoole PW, Nagaraja NV, Hutson AD. 2003. Efficacy of dichloroacetate as a lactate-lowering drug. J Clin Pharmacol 43: 683-91.

57. Ojuka EO. 2004. Role of calcium and AMP kinase in the regulation of mitochondrial biogenesis and GLUT4 levels in muscle. Proc Nutr Soc 63: 
58. Merrill GF, Kurth EJ, Hardie DG, Winder WW. 1997. AICA riboside increases AMP-activated protein kinase, fatty acid oxidation, and glucose Pptake in rat muscle. Am J Physiol 273: E1107-2.

59. Winder WW, Holmes BF, Rubink DS, Jensen EB, et al. 2000. Activation of AMP-activated protein kinase increases mitochondrial enzymes in skeletal muscle. J Apol Physiol 88: 2219-26.

60. Dandapani M, Hardie DG. 2013. AMPK: opposing the metabolic changes in both tumour cells and inflammatory cells? Biochem Soc Trans 1: $687-93$

61. Pilon G, Dallaire P, Marette A. 2004. Inhibition of inducible nitric-oxide synthase by activators of AMP-activated protein kinase: a new mechanism of action of insulin-sensitizing drugs. J Bial Chem 279: 20767-74.

62. Hawley SA, Fullerton MD, Ross FA, Schertzer JD, et al. 2012. Th ancient drug salicylate directly activates AMP-activated protein kinase. Science 336: 918-22.

63. Lee HM, Kim JJ, Kim HJ, Shong M, et al. 2013. Upregulated NLRP3 inflammasome activation in patients with type 2 diabetes. Diabetes 62 194-204

64. Ben Sahra I, Le Marchand-Brustel Y, Tanti JF, Bost F, 2010. Metformin in cancer therapy: a new perspective for an old antidiabetic drug? Mol Cancer Ther 9: 1092-9.

65. Hong J, Zhang Y, Lai S, Lv A, et al. 2013. Effects of metformin versus glipizide on cardiovascular outcomes in patients with type 2 diabetes and coronary artery disease. Diabetes Care 36: 1304-11.
66. Gou S, Cui P, Li X, Shi P, et al. 2013. Low concentrations of metformin selectively inhibit $\mathrm{CD} 133(+)$ cell proliferation in pancreatic cancer and selectively inhibit CD133(+) cell proiferation

67. Pierotti MA, Berrino F, Gariboldi M, Melani C, et al. 2013. Targeting Pierotti MA, Berrino F, Gariboldi M, Melani C, et al. 2013. Targeting
metabolism for cancer treatment and prevention: metformin, an old drug with multi-faceted effects. Oncogene 32: 1475-87.

68. Park CS, Bang BR, Kwon HS, Moon KA, et al. 2012. Metformin reduces Park CS, Bang BR, Kwon HS, Moon KA, et al. 2012. Metformin reduces ainway inflammation and remodeling via activation

69. Nath N, Khan M, Paintlia MK, Singh I, et al. 2009. Metformin attenuated the autoimmune disease of the central nervous system in animal model of multiple sclerosis. $J$ immunal 182: 8005-14.

70. Finlay DK, Rosenzweig E, Sinclair LV, Feijoo-Carnero C, et a 2012. PDK1 regulation of $m T O R$ and hypoxia-inducible factor integrate metabolism and migration of CD8+ T cells. J Exp Med 209: 2441-53

71. Cho DC, Hutson TE, Samlowski W, Sportelli P, et al. 2012. Two phase 2 trials of the novel Akt inhibitor perifosine in patients with advanced rena cell carcinoma after progression on vascular endothelial growth factortargeted therapy. Cancer 118: 6055-62.

72. Bukowski RM. 2012. Temsirolimus: a safety and efficacy review. Exper Opin Drug Saf 11: 861-79.

73. Luo W, Semenza GL. 2011. Pyruvate kinase M2 regulates glucose metabolism by functioning as a coactivator for hypoxia-inducible factor 1 in cancer cells. Oncotarget 2: 551-6. 